\title{
Social-Aware Robot Navigation in Urban Environments
}

\author{
Gonzalo Ferrer, Anaís Garrell and Alberto Sanfeliu
}

\begin{abstract}
In this paper we present a novel robot navigation approach based on the so-called Social Force Model (SFM). First, we construct a graph map with a set of destinations that completely describe the navigation environment. Second, we propose a robot navigation algorithm, called social-aware navigation, which is mainly driven by the social-forces centered at the robot. Third, we use a MCMC Metropolis-Hastings algorithm in order to learn the parameters values of the method. Finally, the validation of the model is accomplished throughout an extensive set of simulations and real-life experiments.
\end{abstract}

\section{INTRODUCTION}

Nowadays, robots are expected to interact naturally in typically human environments. Therefore, urban robots require some basic tools in order to safely being deployed in social environments and accepted by people.

In this paper, we propose a novel robot social navigation for both indoor and outdoor environments. In order to model the social interactions, we use the Social Force Model (SFM) introduced by Helbing [1]. Specifically, this work presents a powerful scheme for robot's human-aware navigation based on the social-forces concept. Moreover, we introduce a new metric, inspired in the classical definition of mechanical work: the social work. This metric serves to evaluate the navigation performance in a social manner.

Robot navigation is a mature field of robotics; there exist many works that demonstrate that robots are able to navigate in challenging environments [2]. However, more socialinteractive approaches are required. Our work is greatly based on Potential Field methods [3], [4] as they keep a great synergy with the social force model, but focusing on the social acceptance. An alternative approach to the SFM, from the learning point of view [5], [6], shed light into the human motion modeling problem.

More recent publications, like [7] deal with urban environments and complex environments, but they consider persons as obstacles while [8] plans a robot navigation in highly crowded environments.

Because a mobile robot must be able to avoid obstacles in the environment where it is working, many different algorithms for obstacle avoidance have been developed. Often, dynamic obstacles are handled only in a locally reactive manner, as static (non-moving). Some works that do account for vehicle kinetics include the Curvature Velocity Method [9] or the Dynamic Window Approach [10]. Other algorithms consider obstacles moving over time [11], [12].

Work supported by the Spanish Ministry of Science and Innovation under project RobTaskCoop (DPI2010-17112).

The authors are with the Institut de Robòtica i Informàtica Industrial (CSIC-UPC). Llorens Artigas 4-6, 08028 Barcelona, Spain. \{gferrer, agarrell, sanfeliu\}@iri.upc.edu

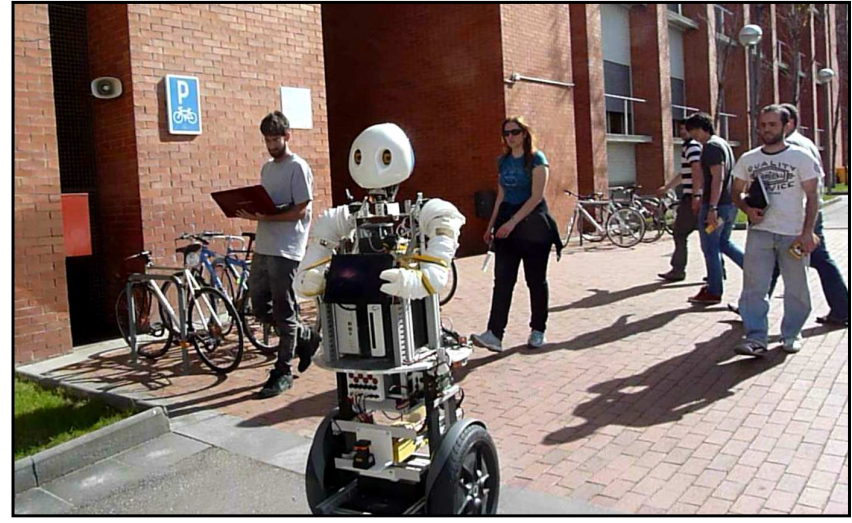

Fig. 1. Social Robot Navigation: Dabo robot navigating in the Barcelona Robot Lab.

Finally, several approaches consider both vehicle dynamics and dynamic obstacles [13], [14]. While all of these algorithms may be used to generate varying degrees of safe and effective obstacle avoidance, none of them explicitly account for the pre-established social conventions that people use when moving around each other.

A number of methods have been developed to allow robots to navigate around people in specific, typically nongeneralizable tasks. Some of these tasks include standing in line [15]; tending toward the right side of a hallway, particularly when passing people [16]; and approaching people to join conversational groups [17]. Museum tour guide robots are often given the capability to detect and attempt to handle people who are blocking their paths [2], [18], [19]. Some researchers have begun researching how a robot might adapt its speed when traveling besides a person, but they have obtained mixed results, even in controlled laboratory settings [20].

Safety and reliability are key factors to the successful introduction of robots into human environments. In most studies, safety is assured by preventing humans from approaching the robots. But said methods are rendered ineffective whenever the robot is designated to directly assist a human individual. In [21], the notion of safety is studied in detail with respect to all relevant aspects of Human-Robot Interaction.

In the present paper, a novel robot social navigation approach based on the so-called social-forces model is introduced, [1]. A model capable of navigating in crowded environments in an acceptable social way is presented.

In the remainder of the paper we start by introducing the theory of the social force model. Section III describes briefly the destination map and the global planner used. Section IV 
presents robot's social-aware navigation, the learning of its parameter and a novel metric to evaluate the social work. Results and conclusions are presented in sections V and VI, respectively.

\section{Social-Force Model}

In order to achieve a model capable of represent the interactions between a pedestrian and a robot, we were inspired by works of Helbing [1] and Zanlungo [22]. Their main contribution is the idea that changes in behavior can be explained in terms of social fields or forces.

Formally, this approach treats each pedestrian $p_{i}$ with mass $m_{i}$ as a particle abiding the laws of Newtonian mechanics:

$\left[\begin{array}{c}x \\ y \\ v_{x} \\ v_{y}\end{array}\right]_{t+1}=\left[\begin{array}{cccc}1 & 0 & \Delta t & 0 \\ 0 & 1 & 0 & \Delta t \\ 0 & 0 & 1 & 0 \\ 0 & 0 & 0 & 1\end{array}\right]\left[\begin{array}{c}x \\ y \\ v_{x} \\ v_{y}\end{array}\right]_{t}\left[\begin{array}{cc}\frac{\Delta t^{2}}{2} & 0 \\ 0 & \frac{\Delta t^{2}}{2} \\ \Delta t & 0 \\ 0 & \Delta t\end{array}\right]\left[\begin{array}{l}a_{x} \\ a_{y}\end{array}\right]$

where $(x, y)$ is person's position, $\left(v_{x}, v_{y}\right)$ is his/her velocity and $\left(a_{x}, y_{x}\right)$ is the acceleration.

Formally, the social forces model assumes that a pedestrian $p_{i}$ with mass $m_{i}$ tries to move at a certain desired speed $v_{i}^{0}$ in a desired direction $\boldsymbol{e}_{i}$, i.e., with desired velocity $\boldsymbol{v}_{i}^{0}=v_{i}^{0} \boldsymbol{e}_{i}$. The desired velocity's direction is given by a vector pointing from the present position of the person $\boldsymbol{r}_{i}$ to the next subgoal $\boldsymbol{g}_{i}$, where the speed is the one at which the human feels more comfortable to walk.

Hence, the basic equation of motion for a pedestrian is given by a social force term:

$$
\frac{d \boldsymbol{v}_{i}(t)}{d t} m_{i}=\boldsymbol{F}_{i}(t)
$$

and describes the movements of the pedestrian $p_{i}$ over time. For the sake of simplicity, we will value $m_{i}$ as the unity for all the persons considered.

A person wants to keep his/her desired velocity through the steering force, $\boldsymbol{f}_{i}{ }^{\text {goal }}$, but is also influenced by others pedestrians $p_{j}, \boldsymbol{f}_{i, j}^{i n t}$, by obstacles, $\boldsymbol{f}_{i, o}^{i n t}$ and, in the present study we model the robot interaction $f_{i, r}^{i n t}$. The resulting force $\boldsymbol{F}_{i}$ governs the trajectory described by the target $p_{i}$.

$$
\boldsymbol{F}_{i}=\boldsymbol{f}_{i}^{\text {goal }}+\boldsymbol{F}_{i}^{i n t}
$$

Below, the description of each component of $\boldsymbol{F}_{i}$ is presented. Assuming that pedestrian tries to adapt his or her velocity within a relaxation time $k_{i}^{-1}, \boldsymbol{f}_{i}^{\text {goal }}$ is given by:

$$
\boldsymbol{f}_{i}^{\text {goal }}=k_{i}\left(\boldsymbol{v}_{i}^{0}-\boldsymbol{v}_{i}\right)
$$

The relaxation time is the interval of time needed to reach the desired velocity and the desired direction.

Furthermore, repulsive effects from the influences of other people, obstacles and robot in the environment are described by an interaction force $F_{i}^{i n t}$. This force prevents humans from walking along their intended direction, moreover, it is modeled as a summation of forces either introduced by

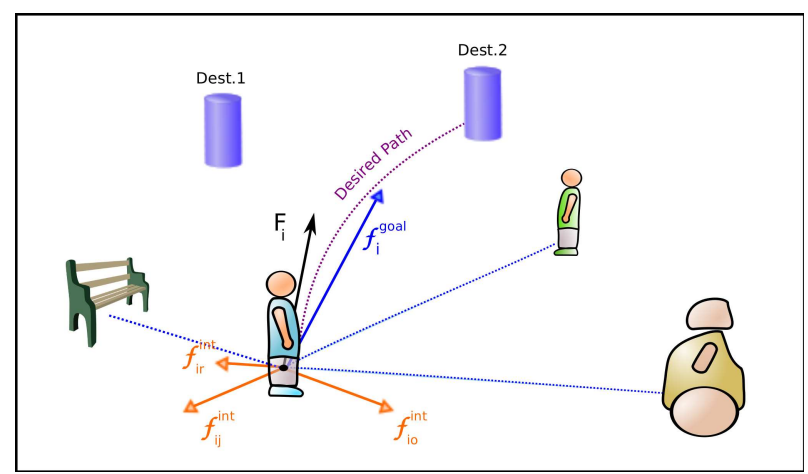

Fig. 2. Diagram of the social forces corresponding to the person $p_{i}$. The blue arrow represents the force aiming to a destination and the orange arrows represent each of the different kinds of interaction forces: personperson, object-person and robot-person. The summation of all the forces is represented as the black arrow $\boldsymbol{F}_{i}$.

people $p_{i}$, by static obstacles in the environment $o$ or the robot $r$. A diagram of the social forces corresponding to the person $p_{i}$ is plotted in Fig. 2.

$$
\boldsymbol{F}_{i}^{i n t}=\sum_{j \in P} \boldsymbol{f}_{i, j}^{i n t}+\sum_{o \in O} \boldsymbol{f}_{i, o}^{i n t}+\boldsymbol{f}_{i, r}^{i n t}
$$

where, $P$ is the set of people moving in the environment where the human interacts and $O$ is the set of obstacles. These forces are modeled as:

$$
\boldsymbol{f}_{i, q}^{i n t}=A_{q} e^{\left(d_{q}-d_{i, q}\right) / B_{q}} \frac{\boldsymbol{d}_{i, q}}{d_{i, q}}
$$

here $q \in P \cup O \cup\{r\}$ is either a person, an object of the environment or the robot. $A_{q}$ and $B_{q}$ denote respectively the strength and range of interaction force, $d_{q}$ is the sum of the radii of a pedestrian and an entity and $\boldsymbol{d}_{i, q} \equiv \boldsymbol{r}_{i}-\boldsymbol{r}_{q}$. In order to calculate the Euclidean distance between $p_{i}$ and the entity $q$, humans and objects are assumed to be of circular shape with radii $r_{i}$ and $r_{q}$. The parameters $A_{q}, B_{q}, d_{q}$ are defined depending on the nature of the object (person, robot or obstacle).

Given the limited field of view of humans, influences might not be isotropic. This is formally expressed by scaling the interaction forces with an anisotropic factor depending on $\varphi_{p, q}$ between $\boldsymbol{v}_{i}$ and $\boldsymbol{d}_{i, q}$

$$
w\left(\varphi_{i, q}\right)=\left(\lambda+(1-\lambda) \frac{1+\cos \left(\varphi_{i, q}\right)}{2}\right)
$$

where $\lambda$ defines the strength of the anisotropic factor,

$$
\cos \left(\varphi_{i, q}\right)=-\boldsymbol{n}_{i, q} \cdot \boldsymbol{e}_{r}
$$

The term $\boldsymbol{n}_{i, q}$ is the normalized vector pointing from $\mathrm{q}$ to person $p_{i}$ which describes the direction of the force.

All these forces are used to define robot's motion, and furthermore, we combine the people tracker with the pedestrian dynamics model to obtain more realistic human motion predictions.

We consider three kinds of interaction forces: personperson, person-obstacle and person-robot. The first and the second interactions has been studied in previous papers 


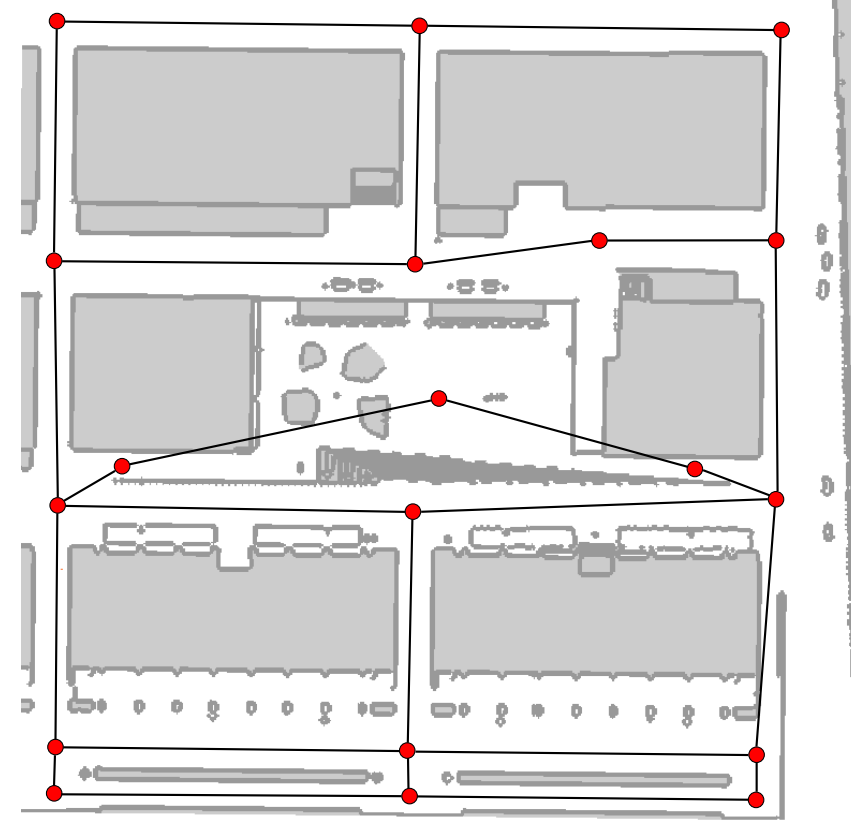

Fig. 3. Map of the Barcelona Robot Lab. Red dots are the set of destinations that describe the navigation environment.

like [1], [22], [23]. The person-robot interaction parameters $\left\{A_{r p}, B_{r p}, \lambda_{r p}, d_{r p}\right\}$ were obtained in [24], specifically for our robotic platform.

\section{GLOBAL MAP}

In this section we propose how to structure the environment in order to build a useful map for a social robotic navigation. To this end, and using the Barcelona Robot lab (although it is generalizable to any other environment), we have obtained manually a set of destinations that completely describe the navigation environment. We consider the following hypothesis: a set of destinations, similar to those goals explained in Sec. II, may be sufficient to describe a global planning in urban environments.

Other classical navigation works like [3] and [4] proposed a similar navigation environment described by attractors. Our approach is not novel in this aspect, however, once obtained a set of these destinations in a urban environment, we will be ready to make use of the social forces to make the robot navigate in a social way, which is one of the main contributions of the present paper.

The implementation of the global planning is a straightforward approach using a search algorithm (in our work we use an $\mathrm{A}^{*}$ implementation) to solve the shortest path from one point of the scene to another (map depicted in Fig. 3).

\section{Social-Aware NAVigation}

Previously, we described a general social interaction model based on social-forces (Sec. II). In this section, we will provide the formulation to build an unified navigation framework using the following idea: the robot is considered as a social agent moving naturally in human environments accordingly to the Social-Force Model, and thus, aiming to a destination and reacting to obstacles and people. Furthermore, we believe that a more humanized navigation, in the sense that the robot responds to the SFM, will highly increase the acceptance over pedestrians, due to the similarities between the robot behavior and the expected behavior of another pedestrians.

To this end, we propose a novel approach to the robot navigation issue, called social-aware navigation, understood as an instantaneous reaction to sensory information, driven by the social-forces centered at the robot. More precisely, we aim to obtain a short-term goal-driven robot navigation ruled by the SFM.

Thereby, it is mandatory to clearly formulate all the social-forces (Sec. II) intervening in the social-aware navigation approach. The following equations are straightforward derivations of the eqs. 3-7. The robot destination is computed in the global planning and the force that drives the robot towards it:

$$
\boldsymbol{f}_{r, \text { dest }}^{\text {goal }}=k_{r}\left(\boldsymbol{v}_{r}^{0}-\boldsymbol{v}_{r}\right)
$$

Once the robot reaches the destination subgoal, the next destination calculated in the global planner becomes the new destination subgoal. The interaction forces due to the pedestrians are the repulsive forces that each person generates to the robot, and they are defined as follows:

$$
\boldsymbol{F}_{r}^{p e r}=\sum_{j \in P} \boldsymbol{f}_{r, j}^{i n t}
$$

where the forces $f_{r, j}^{i n t}$ represent the interaction between the pedestrian $j$ and the robot:

$$
\boldsymbol{f}_{r, j}^{i n t}=A_{r p} e^{\left(d_{r p}-d_{r, j}\right) / B_{r p}} w\left(\varphi_{r, j}, \lambda_{r p}\right)
$$

which is the formulation of the spherical force (Eq. 6) using the parameters $\left\{A_{p r}, B_{p r}, \lambda_{p r}, d_{p r}\right\}$. These parameters correspond to the person-to-robot interaction, and in general are dependent of the robotic platform used.

Correspondingly, the interaction between robot and obstacles is modeled as:

$$
\boldsymbol{F}_{r}^{o b s}=\sum_{o \in O} \boldsymbol{f}_{r, o}^{i n t}
$$

where $f_{r, o}^{i n t}$ is obtained following

$$
\boldsymbol{f}_{r, o}^{i n t}=A_{r o} e^{\left(d_{r o}-d_{r, o}\right) / B_{r o}} w\left(\varphi_{r, o}, \lambda_{r o}\right)
$$

using the specific parameters $\left\{A_{r o}, B_{r o}, \lambda_{r o}, d_{r o}\right\}$ corresponding to the interaction person-obstacle.

Similarly as presented in section II, repulsive effects from the influences of other people and obstacles in the environment are described by an interaction force which is a sum of forces either introduced by people or by static obstacles in the environment.

The combination of the forces described above, which include goal and interacting forces, describes the resultant force governing the robot movement: 


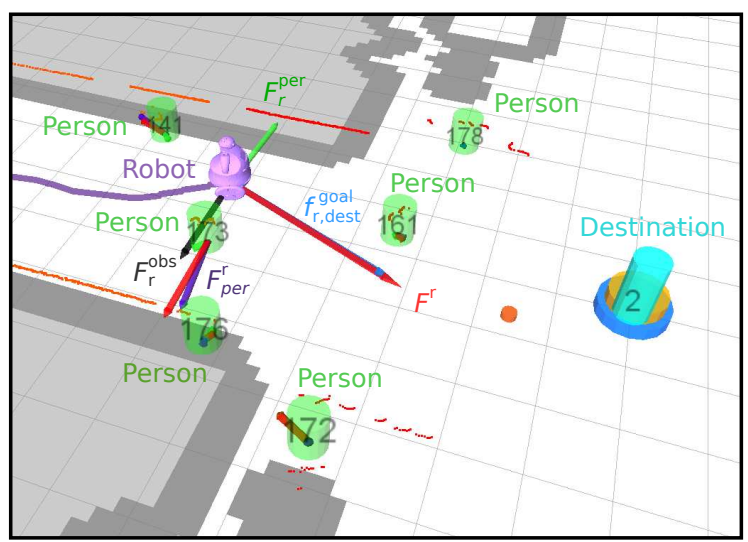

Fig. 4. Robot's Social Forces: Forces applied to the robot while accompanies a person.

$$
\boldsymbol{F}_{r}=\alpha \boldsymbol{f}_{r, \text { dest }}^{\text {goal }}+\gamma \boldsymbol{F}_{r}^{p e r}+\delta \boldsymbol{F}_{r}^{o b s}
$$

Once calculated the resultant social-force, the robot behaves consequently to these external stimuli and propagates its state according to this force value.

Additional constraints are taken into account. All those robot propagations which result in a collision with an obstacle, are forbidden. Current robot maximum velocity is also a constraint and it depends on the robot navigation state, which is a function of the proximity of persons:

$$
v= \begin{cases}v_{\text {safety }} & \text { if } \frac{d_{r, p}}{w\left(\varphi_{r, p}\right)} \leq \mu_{\text {safety }} \\ v_{\text {cruise }} & \text { if } \mu_{\text {safety }}<\frac{d_{r, p}}{w\left(\varphi_{r, p}\right)} \leq \mu_{\text {social }} \\ v_{\text {free }} & \text { otherwise }\end{cases}
$$

The $v_{\text {safety }}$ is the maximum velocity the robot can achieve when at least one person is inside its inner safety zone. We have proposed a social distance to define this region as $d_{r, p} w\left(\varphi_{r, p}\right)$, similarly as described in Sec. II, as a metric of the relative distance between the robot and a pedestrian and an asymmetric factor deforming the distance measure $w\left(\varphi_{r, p}\right)$. This condition also corresponds to the inner robot navigation state. On the other hand, $v_{\text {cruise }}$ is the cruise velocity when someone is inside its social safety zone and $v_{\text {free }}$ is the maximum robot velocity when there are no people inside its safety zone. The navigation states associated to this configurations are the social robot navigation and the free robot navigation, correspondingly. These velocities guarantee that the robotic platform is able to stop according to the resulting forces, before collision occurs.

The most interesting part of the system so far, resides in the fact that the approach proposed is able to navigate near moving persons (dynamic environments) and succesfully reach its goal. The following section discusses the procedure to obtain the value of the parameters $\{\alpha, \gamma, \delta\}$.

\section{A. Parameter Learning}

The computation of the weights of the system parameters, defined as $\theta=\{\alpha, \gamma, \delta\}$, is a mandatory step, prior to the deployment in real environments in a successful and safe way. To this end, we require an initial estimation to learn the magnitude of the $\theta$ parameters.

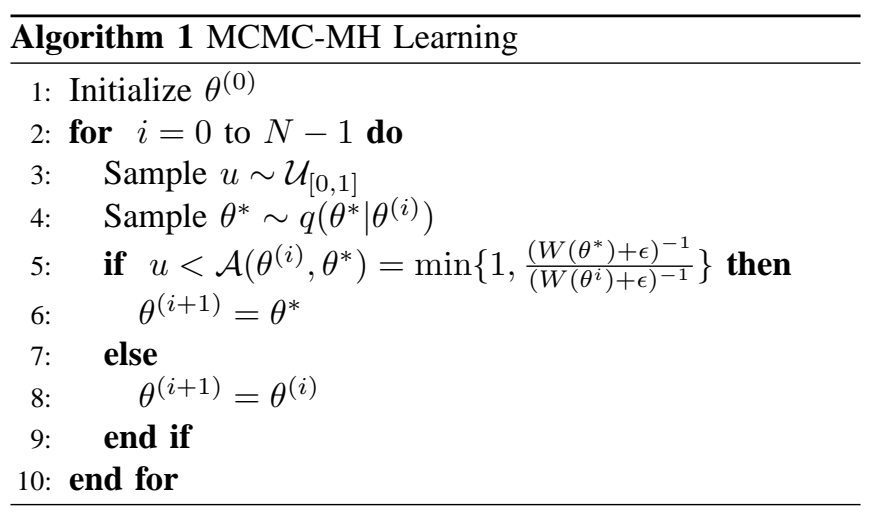

In this case, we will propose a cost function that takes into account the social-forces intervening during the robot navigation. As stated before, we aim to obtain a social robot model capable of dealing with navigation issues in a more human-oriented manner. Consequently, we make use of a variation of the classical definition of work applied to socialforces, similarly to the social work proposed in [25]. The amount of social work corresponding to a time step $\Delta t$ at time $t$ :

$$
W(t, \theta)=W_{r}(t, \theta)+\sum_{i \in P} W_{i, r}(t, \theta)
$$

which represent the social work generated. It consists of the total work done by the robot $W_{r}(t, \theta)=\boldsymbol{F}_{r} \Delta \boldsymbol{s}_{r}$ and the summation of the work done by each person $i$ in the scene, enforced by the robot $W_{i, r}(t, \theta)=\boldsymbol{f}_{i, r}^{i n t} \Delta \boldsymbol{s}_{i}$.

Although the initial conditions can be identically copied throughout all simulations, given the interactive nature of the approach, the parameters $\theta$ alter the outcome $W(\theta)=$ $\sum_{t} W(t, \theta)$ of each experiment (random variable). That is the main reason for considering as an appropriate method for estimating the navigation parameters stochastic optimization. Monte Carlo methods are especially useful for simulating phenomena with significant uncertainty in inputs and systems with a large number of coupled degrees of freedom. More concretely, we have implemented a Markov Chain Monte Carlo Metropolis-Hastings (MCMC-MH) algorithm to find the best set of $\theta$, implementing the Alg. 1 .

The term $q\left(\theta^{*} \mid \theta^{(i)}\right)$, appearing in the algorithm description, represents a Gaussian sampling for each parameter, centered at $\theta^{(i)}$ and a determined variance for each of the variables, which are independent. For stability reasons, $\epsilon$ is a small value that guarantees no singularities in the algorithm, specially when the social work calculated may be zero.

Then, we obtain the best $\hat{\theta}$ parameters as follows:

$$
\hat{\theta}=\arg \min _{\theta}\left\{E_{P(\theta)}\left\{\sum_{t} W(t, \theta)\right\}\right\}
$$

Note that the outcome of the simulations is averaged using the expectation $E_{P(\theta)}\{\}$. 


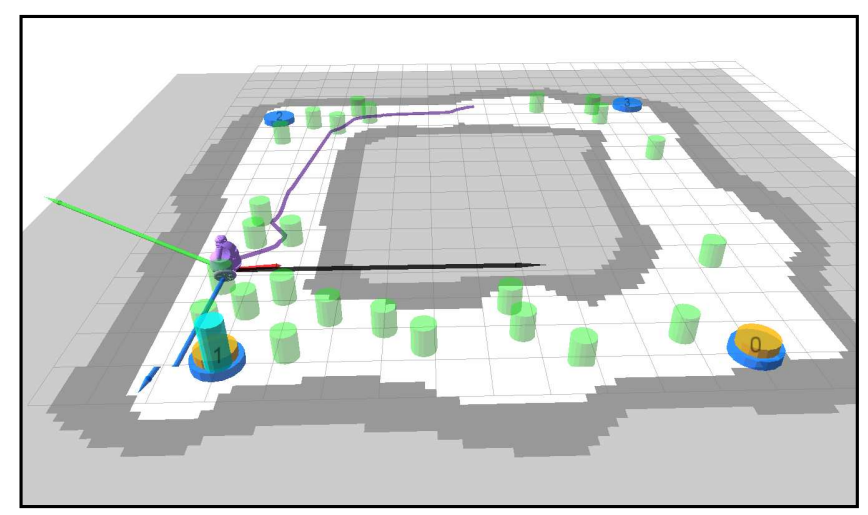

Fig. 5. Simulation experiment: Simulation environment to obtain the $\theta$ parameters. A reduced urban environment and a set of virtual dynamical persons are used. The outcome of each simulations depends on its inner parameters, and thus we required a great number of them.

\section{EXPERIMENTS}

\section{A. Robot and environment description}

In order to conduct all the experiments and to test the approach presented, we have used two twin mobile service robots developed for the URUS project [7], called Tibi and Dabo, designed to work in urban pedestrian areas and interact with people.

They are based on a two-wheeled Segway RMP200 differential platform equipped with two Hokuyo UTM-30LX 2D laser range sensors used to detect obstacles and people.

The experimental area where the experiments are conducted is the BRL (Barcelona Robot Lab), an outdoor urban environments located at the North Campus of the Universitat Politècnica de Catalunya (UPC). The BRL (map in Fig. 3) is a large section of the campus that was outfitted as an experimental area, covering over $10.000 \mathrm{~m}^{2}$, Some pictures of the BRL can be seen in Fig. 6-Top, as well as pedestrians during the robot navigation.

\section{B. Parameter Learning using simulation}

The synthetic scenario used during the simulation step is depicted in Fig. 5, as a simplification of a urban environment. The studied environment consists of static obstacles and multiple people modeled as dynamical obstacles following the SFM, quite similar to a real urban dynamical environment.

The results of the MCMC-MH optimization, explained above in Sec. IV-A, are obtained after more than a thousand simulations. The outcome of each experiment was dependent on the parameters $\theta$, since the system reacts to the behavior of the robot navigation and vice versa. After following the optimization method proposed, we have obtained the values of $\theta$ equal to $\{\alpha=1.0, \gamma=3.18, \delta=0.20\}$, which will be the system parameters that we will use in real experimentation.

\section{Real experiments}

Real experimentation was carried out in a urban environment, the Barcelona Robot Lab, which was described above. The parameters were obtained using simulation learning, as

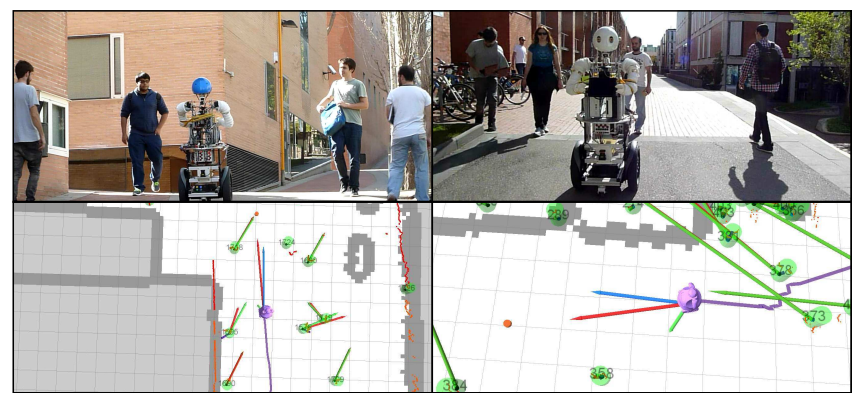

Fig. 6. Real experiments. Top: some pictures of the social-aware navigating in the BRL. Bottom: their corresponding sensor information and relevant information in the robot GUI.

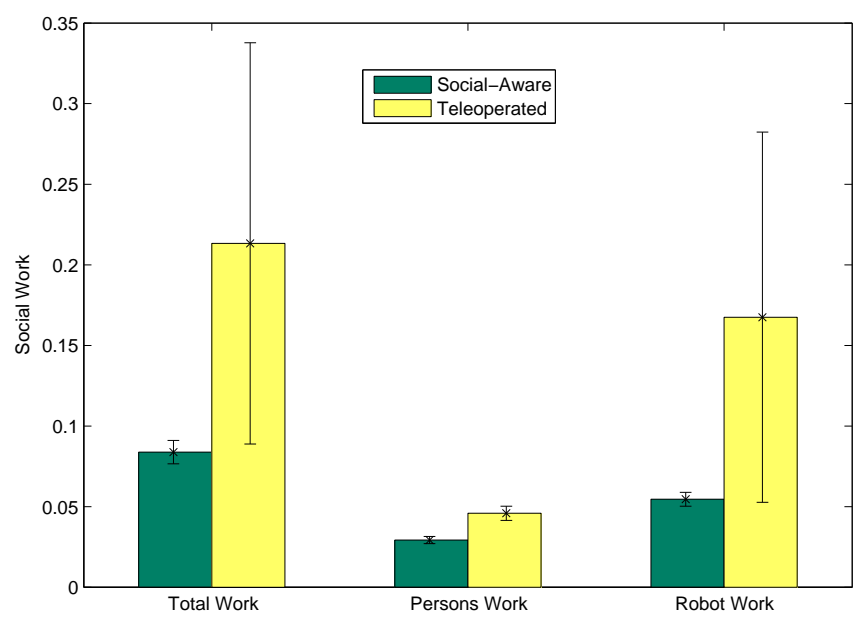

Fig. 7. Experiments results. Average and standard deviation of the social work while performing real experiments. Green bars correspond to the social-aware approach and yellow bars correspond to the tele-operated approach, as a comparison to the proposed method.

stated in the previous section, and during all experiments, those social-aware parameters remained unchanged.

A single experiment consisted in a query of the desired goal to the social-aware navigation and the outcome of the robot navigation corresponds to the path executed in a urban environment with multiple pedestrians and static obstacles. The set of experiments were carried out during a lapse of time equal to two hours, sum in total 20 queries to reach destinations within the BRL (see map in Fig. 3). Almost all navigations commands resulted in goal successfully achieved, except for some problems experimented in narrow passages, were oscillatory problems were detected. In Fig. 6 are depicted some shots of the robot navigating in the BRL, on the top row, and their corresponding sensor information and relevant information in the robot GUI, appearing in the bottom row of the figure.

In order to validate the model in real experiments, we have compared our approach with respect to a tele-operated robot by an expert controller. This experiment was performed under the same conditions as the social-aware navigation: a goal is provided, but instead of an autonomous solution to the navigation, we sought an expert controller to solve the navigation problem, while reaching its goal and dealing with any pedestrian or obstacle on the robot's path.

We have evaluated the performance of both approaches 
using the social work metric proposed in Eq. 16 for the parameter learning. As can be seen in Fig. 7, we have drawn the average and the variance values of the robot's social work, the persons social work enforced by the robot and the total social work of the overall approach. However, the results of the social work were only taken into account if the robot navigation state was social robot navigation or inner robot navigation, that is, if there was at least one person within the social-navigation region, which represent the most interesting cases of study for human interaction purposes.

The social-aware generated less amount of social work both in the robot and the persons surrounding the robot. The comparison of the persons social work for both approaches is similar, both approaches generate a reduced and maybe unavoidable amount of social work. The trivial solution of "escaping" is not an option: this behavior would not solve the interaction with other persons. Accordingly, we evaluate the social work carried out by the robot. In this case, the comparison between our approach and the tele-operated is quite significant, the social-aware approach outperforms the teleoperated approach and its variance is also greatly smaller, which represents more ability to cope more consistently with different situations requiring less social work.

For further information, check the videos of the experimental results and all code in the project web http://www.iri.upc.edu/groups/lrobots/social_aware_ navigation/ecmr2013.php

\section{COnClusions And Future Work}

We have presented a novel robot navigation approach based on the so called Social-Forces Model.

The validation of the model has been demonstrated throughout an extensive set of simulations and real-life experiments in a urban area. In contrast to other existing approaches, our method can handle realistic situations, such as dealing with large environments with obstacles and highly crowded scenes. For that reason, this work can be applied to certain specific real robot applications, for instance, guiding tourists or accompanying professional visitors.

In future work, we aim to obtain more sophisticated robot behavior, for instance, solving the oscillatory problems observed and in general making the robot-aware navigation much more robust.

\section{REFERENCES}

[1] D. Helbing and P. Molnár, "Social force model for pedestrian dynamics," in Physical review. E, 72(2), 1995.

[2] W. Burgard, A. B. Cremers, D. Fox, D. Hähnel, G. Lakemeyer, D. Schulz, W. Steiner, and S. Thrun, "Experiences with an interactive museum tour-guide robot," Artificial intelligence, vol. 114, no. 1, pp. $3-55,1999$.

[3] O. Khatib, "Real-time obstacle avoidance for manipulators and mobile robots," in IEEE Proc. of the international conference on Robotics and Automation, vol. 2, 1985, pp. 500-505.

[4] J. Borenstein and Y. Koren, "The vector field histogram-fast obstacle avoidance for mobile robots," IEEE Transactions on Robotics and Automation, vol. 7, no. 3, pp. 278-288, 1991.

[5] M. Kuderer, H. Kretzschmar, C. Sprunk, and W. Burgard, "Featurebased prediction of trajectories for socially compliant navigation," in Proc. of Robotics: Science and Systems (RSS), 2012.
[6] M. Luber, L. Spinello, J. Silva, and K. O. Arras, "Socially-aware robot navigation: A learning approach," in Intelligent Robots and Systems (IROS), 2012 IEEE/RSJ International Conference on. IEEE, 2012, pp. 902-907.

[7] E. Trulls, A. Corominas Murtra, J. Pérez-Ibarz, G. Ferrer, D. Vasquez, J. Mirats-Tur, and A. Sanfeliu, "Autonomous navigation for mobile service robots in urban pedestrian environments," Journal of Field Robotics, 2011.

[8] P. Trautman, "Probabilistic tools for human-robot cooperation," in Human Agent Robot Teamwork Workshop HRI, 2012.

[9] R. Simmons, "The curvature-velocity method for local obstacle avoidance," in Proceedings of the IEEE International Conference on Robotics and Automation, vol. 4, 1996, pp. 3375-3382.

[10] D. Fox, W. Burgard, and S. Thrun, "The dynamic window approach to collision avoidance," Robotics \& Automation Magazine, vol. 4, no. 1, pp. 23-33, 1997.

[11] B. Kluge, "Recursive agent modeling with probabilistic velocity obstacles for mobile robot navigation among humans," in Proceedings of the IEEE/RSJ International Conference on Intelligent Robots and Systems, vol. 1, 2003, pp. 376-380.

[12] A. Matveev, C. Wang, and A. Savkin, "Real-time navigation of mobile robots in problems of border patrolling and avoiding collisions with moving and deforming obstacles," Robotics and Autonomous Systems, 2012.

[13] A. Foka and P. Trahanias, "Predictive control of robot velocity to avoid obstacles in dynamic environments," in Proceedings of the IEEE/RSJ International Conference on Intelligent Robots and Systems, vol. 1, 2003, pp. 370-375.

[14] E. Owen and L. Montano, "Motion planning in dynamic environments using the velocity space," in Proceedings of the IEEE/RSJ International Conference on Intelligent Robots and Systems, 2005, pp. 28332838.

[15] Y. Nakauchi and R. Simmons, "A social robot that stands in line," Autonomous Robots, vol. 12, no. 3, pp. 313-324, 2002.

[16] V. Olivera and R. Simmons, "Implementing human-acceptable navigational behavior and a fuzzy controller for an autonomous robot," in Proceedings WAF: 3rd Workshop on Physical Agents, Murcia, Spain, 2002, pp. 113-120.

[17] P. Althaus, H. Ishiguro, T. Kanda, T. Miyashita, and H. Christensen, "Navigation for human-robot interaction tasks," in Proceedings of the IEEE International Conference on Robotics and Automation, vol. 2, 2004, pp. 1894-1900.

[18] I. Nourbakhsh, C. Kunz, and T. Willeke, "The mobot museum robot installations: A five year experiment," in Proceedings of the IEEE/RSJ International Conference on Intelligent Robots and Systems, vol. 4, 2003, pp. 3636-3641.

[19] S. Thrun, M. Bennewitz, W. Burgard, A. Cremers, F. Dellaert, D. Fox, D. Hahnel, C. Rosenberg, N. Roy, J. Schulte, et al., "Minerva: A second-generation museum tour-guide robot," in Proceedings of the IEEE International Conference on Robotics and Automation, vol. 3. IEEE, 1999.

[20] E. Sviestins, N. Mitsunaga, T. Kanda, H. Ishiguro, and N. Hagita, "Speed adaptation for a robot walking with a human," in Proceedings of the 2nd ACM/IEEE international conference on Human-robot interaction, 2007, pp. 349-356.

[21] R. Alami, A. Albu-Schaeffer, A. Bicchi, R. Bischoff, R. Chatila, A. De Luca, A. De Santis, G. Giralt, J. Guiochet, G. Hirzinger, et al., "Safe and dependable physical human-robot interaction in anthropic domains: State of the art and challenges," in Workshop on pHRI Physical Human-Robot Interaction in Anthropic Domains, IEEE/RSJ International Conference on Intelligent Robots and Systems. Citeseer, 2006.

[22] F. Zanlungo, T. Ikeda, and T. Kanda, "Social force model with explicit collision prediction," EPL (Europhysics Letters), 2011.

[23] M. Luber, G. Tipaldi, and K. Arras, "Better models for people tracking," in IEEE Proc. of the int. conf. on robotics and automation, 2011.

[24] G. Ferrer, A. Garrell, and A. Sanfeliu, "Robot companion: A socialforce based approach with human awareness-navigation in crowded environments," in Proceedings of the IEEE/RSJ International Conference on Intelligent Robots and Systems, 2013.

[25] A. Garrell and A. Sanfeliu, "Cooperative social robots to accompany groups of people," IJRR, 2012. 